> L'exercice physique ou l'hypertension artérielle sont deux situations, l'une physiologique, l'autre pathologique, au cours desquelles le cœur augmente son travail hémodynamique. Cette adaptation repose sur un remodelage cardiaque différent selon la nature physiologique ou pathologique du stress. Illustrée par deux exemples, l'étude des événements moléculaires aboutissant au remodelage cardiaque offre de nouvelles opportunités pour le développement de thérapies de l'insuffisance cardiaque. Récemment décrite, la protéine Epacl est un relais du second messager AMPc. À la suite d'un stress pathologique, la mise en évidence de ses rôles dans l'hypertrophie, la fibrose cardiaque et l'altération du cycle calcique suggère que son inhibition pharmacologique peut présenter un intérêt thérapeutique. Carabin est une nouvelle protéine régulatrice de plusieurs effecteurs moléculaires impliqués dans le remodelage cardiaque pathologique. La manipulation expérimentale de son expression modifie profondément le développement de l'insuffisance cardiaque. <

Le cœur est un organe plastique : sous l'influence de diverses conditions physiologiques ou pathologiques, sa structure et sa fonction s'adaptent au niveau de l'organe lui-même, du tissu et des cellules le composant. L'ensemble de ces modifications sont regroupées sous le terme de remodelage cardiaque dont on distingue deux formes en fonction du stimulus déclenchant et des conséquences en termes de santé.

Chez l'adulte, le remodelage cardiaque physiologique est observé chez le sportif régulier et la femme enceinte. Il se caractérise par une croissance harmonieuse du cœur dans le sens où l'hypertrophie des cardiomyocytes s'accompagne d'un accroissement de leur capacité contractile et d'une adaptation de leur cycle calcique et de leur métabolisme, rendant possible une augmentation

\section{Les acteurs \\ moléculaires \\ du remodelage \\ cardiaque \\ pathologique}

Exemple des protéines

Epac et carabin

\section{Yannis Sainte-Marie ${ }^{1-3}$, Malik Bisserier ${ }^{1,2}$, Florence Tortosa ${ }^{1,2}$, Frank Lezoualc' ${ }^{1,2}$}

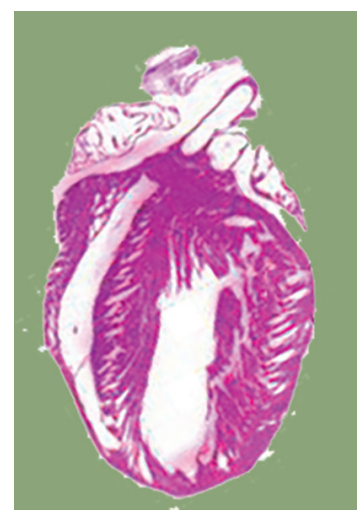

${ }^{1}$ Inserm, UMR-1048, institut des maladies métaboliques et cardiovasculaires,

1 , avenue Jean Poulhès, BP 84225, F-31342 Toulouse Cedex 4, France ;

${ }^{2}$ Université Toulouse III Paul Sabatier, F-31342 Toulouse, France :

${ }^{3}$ Faculté des sciences pharmaceutiques, Université Toulouse III Paul Sabatier, F-31342 Toulouse, France. frank.lezoualch@inserm.fr

du travail cardiaque [1]. Ce remodelage permet de prendre en charge l'augmentation de la perfusion des organes nécessaire dans ces conditions physiologiques.

Le remodelage cardiaque pathologique est observé en association avec différentes pathologies, familiales ou acquises, dont les plus répandues sont l'hypertension artérielle, l'infarctus du myocarde et les troubles du métabolisme. La qualification de pathologique du remodelage, initialement sans conséquences cliniques, repose sur son association avec la survenue ultérieure de pathologies cardiovasculaires. Le remodelage pathologique comprend de multiples atteintes dont les mieux décrites sont la modification de la géométrie de la cavité cardiaque associée à une hypertrophie des cellules contractiles cardiaques (les cardiomyocytes), en particulier dans le sens longitudinal, la fibrose et l'altération de la force contractile cardiaque provenant de modifications diverses du couplage excitation-contraction [2] (Figure 1). Il est à noter que des modifications d'autres tissus (artériels, nerveux, musculaires ou ceux impliqués dans le métabolisme) peuvent participer au développement de l'insuffisance cardiaque (IC), mais nous n'abordons ici que le remodelage du tissu cardiaque. Ces altérations concourent à la mise en place du syndrome clinique d'insuffisance cardiaque lorsque le fonctionnement du cœur n'assure plus la perfusion adéquate des organes $[41](\rightarrow)$.

$(\rightarrow)$ Voir la synthèse de F. Pinet et

C. Bauters, $m / s n^{\circ} 8-9$, août-septembre 2015, page 770

Le remodelage cardiaque pathologique a été identifié comme une cible thérapeutique potentielle à partir d'une étude prospective 


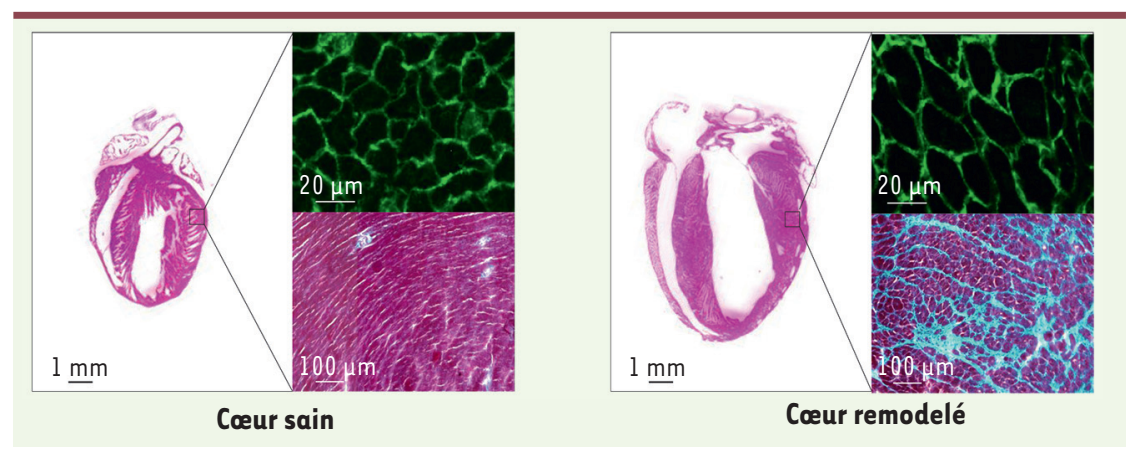

Figure 1. Coupes longitudinales d'un cœur de souris sain (A) et remodelé après une sténose aortique (B). La coupe longitudinale cardiaque, colorée à l'hémalun éosine, montre une dilatation du ventricule gauche dans le cœur remodelé. L’hypertrophie des cardiomyocytes est révélée par microscopie à fluorescence après marquage des membranes des cardiomyocytes à la vinculine (vert). Tout comme l'hypertrophie, la fibrose cardiaque (visualisée en bleu après une coloration au trichrome de Masson) est fortement augmentée dans le cœur remodelé.

réalisée sur la population de Framingham ${ }^{1}$ où l'un des paramètres du remodelage cardiaque, l'hypertrophie, a été associé à la survenue ultérieure de pathologies cardiovasculaires [3]. Cette hypothèse a été confirmée par l'efficacité des traitements actuels qui visent à réduire les contraintes hémodynamiques en contrant la suractivation des systèmes neurohumoraux (catécholamines, rénine-angiotensine-aldostérone). Cette stratégie thérapeutique freine aussi le remodelage cardiaque pathologique de manière décisive [4].

Cependant, les mécanismes qui relaient le stimulus pathologique et conduisent au phénotype délétère restent à découvrir.

\section{Intérêts de la « dissection moléculaire 》 du remodelage cardiaque}

L'insuffisance cardiaque est la conséquence non seulement d'une inadéquation entre les capacités de pompage du cœur et les contraintes hémodynamiques, mais aussi d'un remodelage cardiaque orchestré par un programme génique. Ce point essentiel, à l'origine des recherches actuelles, a été illustré par les travaux pionniers des docteurs Ketty Schwartz, Bernard Swynghedauw et de leurs collaborateurs, qui ont mis en évidence la réexpression d'un programme génique, dit fotal, par le ventricule après un stimulus pathologique [5]. Avec le développement de modèles animaux génétiquement modifiés, l'origine potentiellement intrinsèque du remodelage cardiaque a pu être démontrée. II faut citer ici les travaux fondateurs de Molkentin et Olson portant sur la voie de signalisation de la calcineurine (CaN) [6] (Figure 2). Cette phosphatase, initialement identifiée dans les lymphocytes, est responsable de la translocation nucléaire du facteur de transcription NFAT (nuclear factor of activated T-cells) qui peut ensuite activer ses

${ }^{1}$ Framingham est une ville du Massachusetts intégrée depuis 1948 dans une étude sur les maladies cardiovasculaires. Cette localité a été choisie par l'Institut national du cœur, des poumons et du sang (NHLBI) de Bethesda pour sa représentativité de la population américaine. gènes cibles (voir Figure 3). La surexpression cardiaque de la CaN ou de NFAT chez la souris induit un remodelage cardiaque délétère qui se caractérise, entre autres, par une hypertrophie cardiaque (HC) et une fibrose conduisant à l'insuffisance cardiaque et la mort des animaux. En plus d'identifier la voie CaN/NFAT comme un acteur majeur du remodelage cardiaque, cette étude a montré qu'en l'absence de modification des contraintes hémodynamiques, une seule voie de signalisation était suffisante pour induire un remodelage pathologique. Parmi les différentes cascades de transduction répondant aux stimulus pathologiques, la voie de la CaMKII $\left(\mathrm{Ca}^{2+} /\right.$ calmodulin-dependent protein kinase II) joue un rôle essentiel dans la mise en place du remodelage délétère [7] (Figure 2). Cette kinase, sensible au calcium, est responsable de l'activation du facteur de transcription MEF2 (myocyte enhancer factor 2). Elle lève la répression génique en provoquant la phosphorylation et l'export nucléaire des histone déacétylases (HDAC) (Figure 2).

Le remodelage cardiaque pathologique regroupe des altérations touchant les cardiomyocytes mais également des modifications phénotypiques d'autres types cellulaires peuplant le tissu cardiaque. La multiplication et la transformation des fibroblastes en myofibroblastes participent activement à l'excès de dépôt de matrice extracellulaire. Des altérations des cellules vasculaires sont également impliquées dans une dysfonction coronaire dont la raréfaction capillaire est une conséquence. Les cellules immunitaires interviennent aussi en coordonnant une réponse inflammatoire et en influençant, par les cytokines qu'elles sécrètent, les fonctions des autres types cellulaires cardiaques. II est intéressant de noter que, dans certains modèles expérimentaux, l'hypertrophie des cardiomyocytes (ou la fibrose seule) ne permet pas d'anticiper l'atteinte fonctionnelle du myocarde. Ainsi, l'hypertrophie accrue des cardiomyocytes dans un modèle de surexpression de la NOS1 (nitric oxide synthase 1) soumis à une surcharge de pression n'est pas associée à un dysfonctionnement majoré mais au contraire à un maintien de la fonction cardiaque [8]. Par ailleurs, l'inhibition, par traitement avec un anticorps neutralisant le TGF $\beta$ (transforming growth factor $\beta$ ), de la fibrose induite par une surcharge de pression n'améliore pas la fonction cardiaque mais au contraire augmente la dilatation ventriculaire 


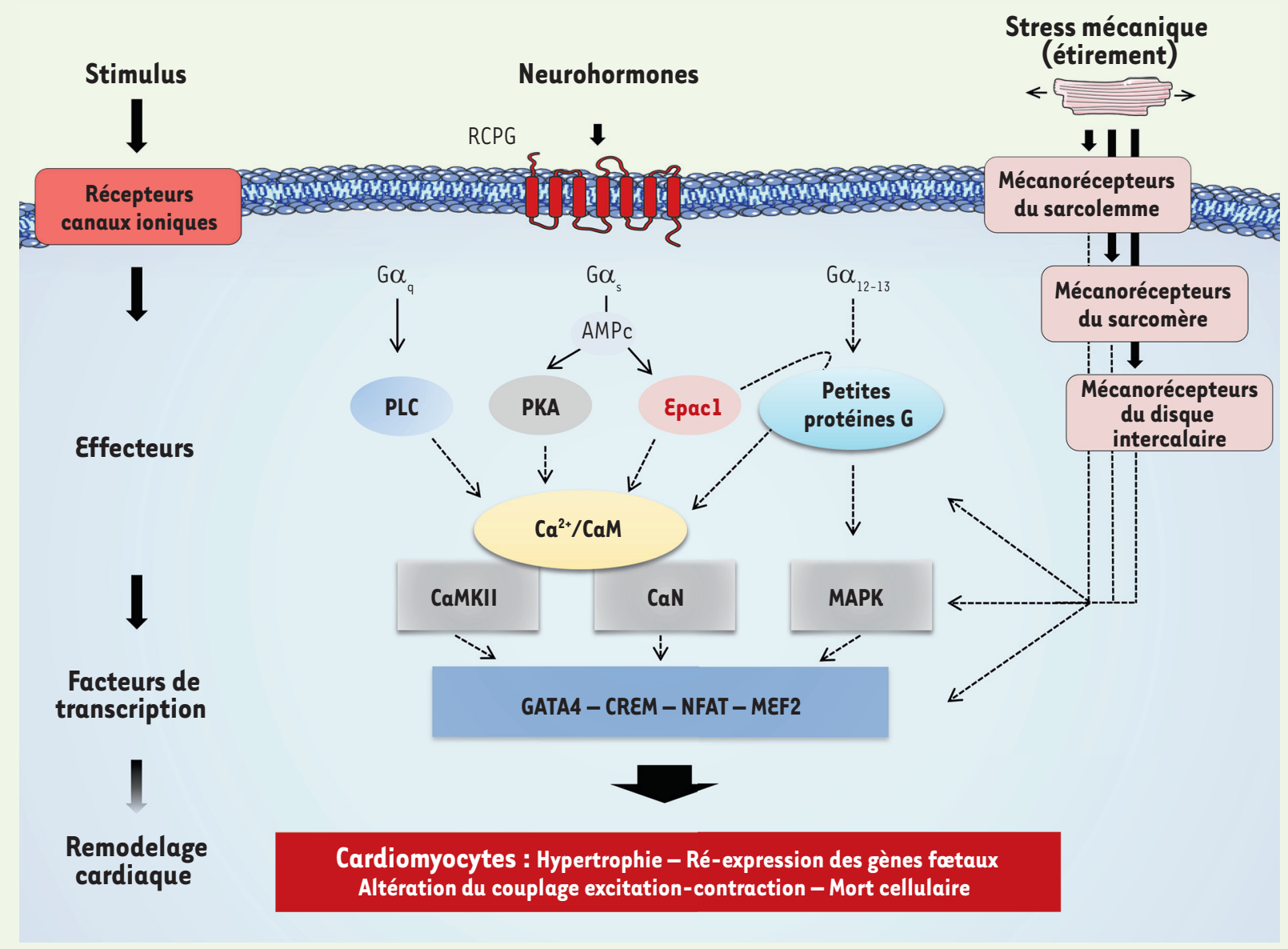

Figure 2. Principales voies de signalisation impliquées dans le remodelage cardiaque pathologique. Les stress neurohumoraux (NE, દT-1, Ang-II) sont transmis par différents types de récepteurs et canaux au sein des cardiomyocytes. Ces récepteurs peuvent être couplés aux protéines $G$ de type $\mathrm{G} \alpha q$, $G \alpha$ s ou $G \alpha_{12 / 13}$ pour activer la phospholipase C (PLC), l'adénylate cyclase et les petites protéines $G$. Les effecteurs en aval impliquent la calcineurine ( $\mathrm{CaN}$ ), la CaMKII et les MAPK. Ces voies de signalisation modulent l'activité de facteurs de transcription en faveur d'un remodelage cardiaque pathologique. Les stress mécaniques sont transmis par des protéines liées aux filaments des sarcomères ou aux complexes de jonctions intercellulaires (disques intercalaires) ou cellule-matrice (sarcolemme). La signalisation associée est méconnue mais les implications de la PKC, de CaN ou de GATA4 sont possibles [40]. $\mathrm{Ca}^{2+} / \mathrm{CaM}: \mathrm{Ca}^{2+} /$ calmoduline ; CaMKII : $\mathrm{Ca}^{2+} /$ calmodulin-dependent protein kinase II; CaN : calcineurine ; CREM : cAMP-responsive element modulator ; Epacl : exchange protein directly activated by cAMP ; MEF2 : myocyte-enhancer factor 2 ; PKA : protéine kinase dépendante de l'AMPC; PLC : phospholipase C; MAPK : mitogen-activated protein kinase; NFAT : nuclear factor of activated T-cells ; $\mathrm{RCPG}$ : récepteur couplé aux protéines G ; NE : norépinéphrine ; $\varepsilon T-1$ : endothéline 1 ; Ang-II : angiotensine II..

et le dysfonctionnement [9]. Ces deux exemples illustrent le fait que le caractère pathologique du remodelage ne se comprend in fine qu'avec l'analyse de l'ensemble des aspects du remodelage et de leurs conséquences fonctionnelles sur le cœur. La communauté scientifique est engagée dans l'étude moléculaire des événements de signalisation liant les stimulus pathologiques et le programme génique de remodelage délétère. Cette «dissection moléculaire » du remodelage, aussi bien pathologique que son alternative physiologique, devrait permettre de faire émerger de nouvelles options thérapeutiques $[10,11]$.

Nous souhaitons donner ici deux exemples issus des recherches menées dans notre laboratoire, et portant sur la signalisation pathologique des cardiomyocytes. Le premier exemple concerne la protéine Epacl (exchange protein directly activated by $c A M P)$. Cette protéine intervient en aval de l'activation des récepteurs $\beta$-adrénergiques ( $\beta-A R$ ), point de divergence conduisant à plusieurs voies de signalisation dont la dissection moléculaire permettra peut-être de développer des stratégies thérapeutiques encore plus efficaces que les $\beta$-bloquants ${ }^{2}$. Le deu-

${ }^{2}$ Les $\beta$-bloquants sont des médicaments qui agissent par antagonistes compétitifs des catécholamines (épinéphrine, norépinéphrine) au niveau des récepteurs $\beta$-adrénergiques. Ils sont par exemple utilisés pour améliorer la contractilité cardiaque. 


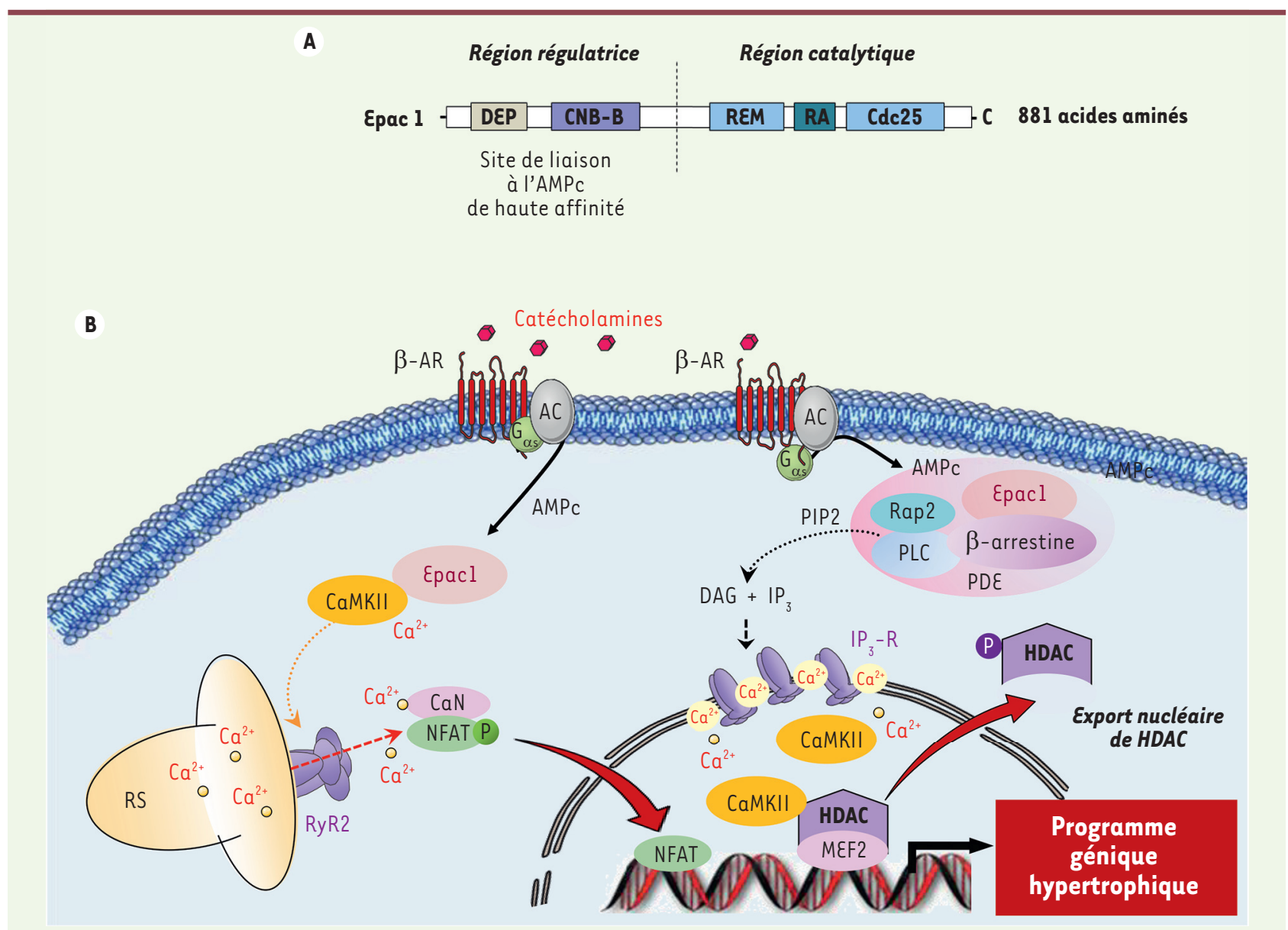

Figure 3. Voies de signalisation associées à Epacl conduisant à l'hypertrophie cardiaque. A. Représentation schématique de la structure de Epacl. L'isoforme Epacl, constituée de 881 acides aminés, présente une région catalytique située en carboxy-terminal, responsable de l'activité d'échange, et une région régulatrice en amino-terminal contenant le site de fixation à l'AMPc. CNB-B : cyclic nucleotide binding domain B ; DEP : dishevelled, Egl-10, Pleckstrin domain; RA : Ras-associating domain; REM : ras exchange motif. B. Représentation schématique de la signalisation associée à Epacl et conduisant à l'hypertrophie. Suite à la stimulation des $\beta$-AR par les catécholamines, Epacl est recrutée à la membrane plasmique par le biais de la $\beta$-arrestine pour activer une voie de signalisation dépendante de la petite protéine G Rap2 et de la phospholipase C (PLC). Les phosphodiestérases (PDE) contrôlent l'activité de Epacl en régulant les concentrations locales d'AMPc. L'activation des récepteurs à l'IP ${ }_{3}\left(I_{3}{ }^{-}\right.$ R) provoque ensuite une augmentation du calcium nucléaire qui active la CaMKII. Une fois phosphorylées par la CaMKII, les HDAC sont exportées du noyau, permettant ainsi à MEF2 d'activer ses gènes cibles. La phosphorylation de RyR2 par la CaMKII induit une fuite calcique au niveau du réticulum sarcoplasmique (RS) pour activer la voie CaN/NFAT et amplifier le programme hypertrophique. AC : adénylyl cyclase ; $\beta$-AR : récepteur $\beta$-adrénergique ; CaMKII : $\mathrm{Ca}^{2+} /$ calmodulin-dependent protein kinase ; $\mathrm{CaN}$ : calcineurine ; DAG : diacylglycérol ; Epacl : exchange protein directly activated by $c A M P ; I_{3}-R: I_{3}$ récepteur ; MEF2 : myocyte-enhancer factor 2 ; NFAT : nuclear factor of activated T-cells; PLC : phospholipase C; PDE : phosphodiestérase ; RS : réticulum sarcoplasmique.

xième exemple est la protéine carabin. Elle se place juste en amont de la convergence nucléaire de la signalisation de la CaMKII, de la calcineurine ou des MAPK (Figure 2). En effet, même si l'inhibition de la calcineurine a été identifiée directement ou indirectement comme une stratégie prometteuse dans des modèles animaux, les molécules pharmacologiques disponibles ne présentent pas un rapport bénéfice/risque favorable. Une stratégie alternative plus spécifique du cardiomyocyte et/ou une meilleure efficacité de ces molécules seraient donc souhaitables.

\section{Epacl : un facteur d'échange favorisant I'hypertrophie pathologique}

\section{Signalisation du second messager AMP cyclique (AMPc)}

L'augmentation des catécholamines et l'activation de leurs récepteurs sont associées cliniquement et expérimentalement à l'induction d'un remodelage cardiaque pathologique [12]. Pourtant, l'utilisation 
des $\beta$-bloquants dans l'insuffisance cardiaque peut paraître contreintuitive puisque les catécholamines représentent le principal stimulant de la contractilité et de la relaxation cardiaques. En effet, ces médicaments resensibilisent le système de signalisation adrénergique qui est désactivé par les concentrations élevées et chroniques de catécholamines [13]. Cela permet de rétablir la réserve inotrope et s'accompagne d'une baisse du remodelage pathologique $[14,15]$. Ainsi, ce n'est pas tant de bloquer l'ensemble de la signalisation adrénergique qui semble important, mais bien de moduler ses différents aspects. En termes de signalisation, les $\beta$-AR sont responsables de multiples effets. Pour citer quelques exemples : production d'AMPc activant la protéine kinase $A M P c$ dépendante (PKA) mais également des facteurs de transcription CREM ( $C A M P$ response element modulator) ; augmentation du calcium en tant que second messager [16, 17]. Ainsi, comprendre l'ensemble des voies en aval des $\beta$-AR semble important pour réussir à améliorer le traitement de l'insuffisance cardiaque. C'est dans ce contexte que nous nous intéressons aux effets cardiaques de nouvelles protéines de liaison à l'AMPc appelées Epac (exchange proteins directly activated by cAMP).

\section{Les protéines Epac}

Les protéines Epac sont des facteurs d'échange directement activés par l'AMPc qui favorisent la forme active des petites protéines $G$ de la famille Ras, Rapl (Ras-associated protein-1) et Rap2 en catalysant l'échange du GDP pour le GTP $[18,19]$. II en existe deux isoformes, Epacl et Epac2, qui sont codées respectivement par les gènes RAPGEF3 (Rap guanine nucleotide exchange factor3) et RAPGEF4. Epacl est fortement exprimée dans le cœur, les vaisseaux sanguins et le rein, tandis que Epac2 a été initialement mise en évidence dans le pancréas et le cerveau [18]. Les protéines Epac présentent une forte homologie de structure. Elles sont constituées d'une région catalytique située du côté carboxy-terminal et d'une région régulatrice en amino-terminal (Figure 3A). La région catalytique permet l'échange du GDP par le GTP sur les petites protéines $G$ effectrices de Epac. La région régulatrice est constituée d'un domaine de liaison à I'AMPc. La fixation de I'AMPc à Epac induit un changement de sa conformation qui libère la contrainte stérique exercée par la région régulatrice sur la région catalytique, activant la protéine [20].

\section{Epacl et remodelage pathologique cardiaque}

La preuve initiale que Epacl est impliquée dans l'hypertrophie des myocytes cardiaques a été obtenue à l'aide de cultures primaires de cardiomyocytes de rats nouveau-nés, un modèle de choix pour identifier les facteurs de remodelage et leurs mécanismes d'action. Ainsi, l'activation directe de Epacl par un analogue de l'AMPc qui lui est spécifique, le 8-pCPT ${ }^{3}$ [21], ou la surexpression de Epacl, favorisent l'hypertrophie cellulaire et l'expression des marqueurs du remodelage cardiaque délétère (programme génique fœtal) [22, 23]. À l'inverse, l'inhibition pharmacologique de Epacl par un analogue de la tétrahydroquinoline, le CE3F4,

${ }^{3}$ Le 8-pCPT-2'-0-Me-cAMP (8-[4-chlorophenylthio]-2'-0-methyladenosine-3', $5^{\prime}$-cyclic monophosphate) est un activateur fort d'Epac. ou l'extinction de Epacl par l'utilisation de shARN, préviennent l'hypertrophie des cardiomyocytes induite par la stimulation des $\beta$-AR [23-25]. Le mécanisme par lequel Epacl provoque l'hypertrophie pathologique des cardiomyocytes n'est pas complètement élucidé. Cependant, des expériences montrent que ce facteur d'échange influence les mouvements calciques intracellulaires par le biais de la phospholipase $C$ et des récepteurs à I'IP $_{3}$ (inositol triphosphate), pour activer les voies de signalisation dépendantes de la phosphatase $\mathrm{CaN}$ et de la CaMKII $[23,26]$. Ces événements moléculaires font intervenir la $\beta$-arrestine, protéine d'échafaudage, et des régulations épigénétiques qui dépendent des HDAC (Figure 3B) [26-28].

L'étude de souris invalidées pour les gènes $\varepsilon p a c l$ et $\varepsilon p a c 2$ a permis de mieux comprendre le rôle de ces protéines dans le remodelage pathologique. Les souris $\varepsilon p a c l^{-/-}$et

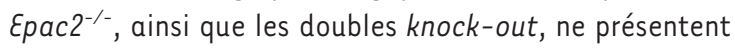
aucune anomalie cardiaque. Ces isoformes ne semblent donc pas indispensables au développement du myocarde et au maintien de la fonction cardiaque à l'état basal [29-31]. Seule la délétion de Epacl réduit le remodelage pathologique induit par l'activation chronique des $\beta-A R$, ce qui confirme l'importance de Epacl dans la signalisation $\beta$-adrénergique au cours de l'hypertrophie cardiaque pathologique. Toutefois, dans un autre modèle d'hypertrophie cardiaque induite par une sténose aortique, l'absence de Epacl ne prévient pas l'hypertrophie cardiaque, mais seulement la fibrose et l'apoptose des cardiomyocytes, ce qui suggère que les effets cardioprotecteurs de Epacl vis-à-vis de l'hypertrophie cardiaque dépendent de la nature du stress [30].

\section{Epacl et fonction cardiaque}

De façon très intéressante, en réponse à différents stress hypertrophiques (injection de catécholamines ou surcharge de pression), les souris $\varepsilon \mathrm{pacl}^{-/-}$présentent une meilleure contractilité cardiaque (maintien de la réserve inotrope) $[30,31]$. Par ailleurs, ces souris sont protégées de la survenue d'arythmies atriales [30]. Ces résultats suggèrent que Epacl participe aux dérèglements calciques lors du remodelage cardiaque pathologique et des arythmies qui lui sont associées (Figure 3B). Ainsi, dans les cardiomyocytes ventriculaires de rat, l'activation de Epacl induit une augmentation de la libération spontanée de calcium à partir des stocks intracellulaires (un phénomène appelé bouffées calciques ou $\mathrm{Ca}^{2+}$ sparks) par le biais d'une hyperphosphorylation des canaux récepteurs de la ryanodine ${ }^{4}$, RyR2 [32], ce qui provoque une fuite calcique

\footnotetext{
${ }^{4}$ La ryanodine est un alcaloïde qui modifie le fonctionnement de certains canaux calciques.
} 
du réticulum sarcoplasmique [33, 34]. II a été proposé que l'augmentation du relargage ectopique de calcium, consécutive à l'activation de Epac par les $\beta$-AR, pourrait être à l'origine des effets arythmogènes dans les cardiomyocytes isolés de souris. Cependant, l'isoforme de Epac impliquée dans ces troubles du rythme reste encore controversée $[29,30,35]$. Une étude récente montre que Epacl accélère le repompage du $\mathrm{Ca}^{2+}$ dans le réticulum sarcoplasmique par la SERCA2 (sarco/ endoplasmic reticulum $\mathrm{Ca}^{2+}$-ATPase) en favorisant I'hyperphosphorylation du phospholamban ${ }^{5}$ par la PKCE (protéine kinase $C \varepsilon$ ). Ceci pourrait induire une surcharge du réticulum sarcoplasmique, propice aux fuites calciques [30]. L'ensemble de ces résultats suggèrent qu'en normalisant les mouvements calciques et en freinant le remodelage cardiaque, un inhibiteur pharmacologique de Epacl pourrait s'avérer efficace pour le traitement de l'insuffisance cardiaque [25].

\section{Carabin : une protéine inhibant l'hypertrophie pathologique}

Au cours de ces dernières années, il est apparu de plus en plus évident que le myocarde possède une variété de mécanismes de rétrocontrôle pour contrebalancer la réponse hypertrophique. À titre d'exemple, l'effet hypertrophique de Epacl induit des processus d'adaptation et de survie pour prévenir le stress cardiaque [31]. La surexpression cardiaque des protéines AKAP79 (A kinase anchor protein), Cabinl/Cain ou PICOT s'oppose à l'hypertrophie cardiaque induite par un stress mécanique ou catécholaminergique [36]. Ces protéines cardioprotectrices ciblent essentiellement la voie de la CaN/NFAT, ce qui en fait tout l'intérêt. Elles peuvent en effet offrir des perspectives thérapeutiques contre l'insuffisance cardiaque.

Nous avons identifié dans le myocarde une autre protéine appelée carabin qui est capable d'interagir non seulement avec la $\mathrm{CaN}$ mais aussi avec la petite GTPase Ras et la CaMKII, deux autres voies de signalisation majeures impliquées dans le remodelage pathologique.

\section{Carabin et remodelage cardiaque}

Carabin fut initialement caractérisée dans le système immunitaire [37]. Cette protéine de 446 acides aminés possède deux régions importantes: une région (séquence 89-294) Ras/Rab GAP (GTPase activating protein) et une région (séquence 406-446) de liaison à la $\mathrm{CaN}$ dans sa partie carboxy-terminale. Dans les cellules $T$ et $B$, carabin inhibe l'activité phosphatase de la CaN et la signalisation associée à la petite protéine G, Ras et son effecteur ERK (extracellular signalregulated kinase) $[37,38]$. Étant donné les rôles critiques joués par ces protéines de signalisation dans la mise en place du remodelage délétère, nous avons émis l'hypothèse que carabin pourrait avoir un rôle cardioprotecteur.

Nos premières expériences explorant l'implication potentielle de carabin dans la régulation de l'hypertrophie cardiaque pathologique ont révélé l'existence d'une corrélation inverse entre l'expression cardiaque de carabin et la sévérité du remodelage cardiaque. Ainsi, nous avons

${ }^{5}$ le phospholamban est une protéine de la membrane du réticulum sarcoplasmique exerçant un effet inhibiteur sur la pompe à calcium SERCA2. constaté que le niveau d'expression de carabin est diminué dans le myocarde de rat et de souris lorsque celui-ci est hypertrophié en réponse à une sténose aortique, mais également dans le cœur des patients atteints d'insuffisance cardiaque [39]. Des études de surexpression et de délétion nous ont permis d'étudier plus finement l'implication de carabin dans la physiopathologie de l'insuffisance cardiaque. La restauration de l'expression cardiaque de carabin, par une approche de transfert de gène in vivo chez la souris, prévient le remodelage et la dysfonction cardiaque induite par une surcharge de pression. De même, in vitro, la surexpression de carabin bloque la réexpression du programme génique fœtal, ainsi que l'hypertrophie des cardiomyocytes, en réponse à des stimulus neurohumoraux. À l'inverse, les souris déficientes en carabin sont hypersensibles à un stress hémodynamique et développent rapidement des anomalies cardiaques menant à une hypertrophie cardiaque et une insuffisance cardiaque [39].

\section{Carabin : une protéine aux cibles multiples}

Au niveau moléculaire, les mécanismes de l'action cardioprotectrice de carabin sont associés à l'inhibition simultanée de trois voies de signalisation qui jouent un rôle déterminant dans l'hypertrophie cardiaque. Carabin inhibe les voies dépendantes de la CaN, de la CaMKII et des petites protéines $G$ Ras par l'intermédiaire de ses domaines d'interaction avec la CaN et Ras-GAP. En effet, la surexpression de carabin dans des cardiomyocytes en culture inhibe l'activité phosphatase de la CaN ainsi que l'activité transcriptionnelle de son effecteur NFAT lors de l'hypertrophie. Par des expériences de mutagenèse dirigée, nous avons montré que carabin réprime, non seulement l'activité de la petite protéine $G$ Ras grâce à son domaine Ras-GAP, mais aussi l'activation de la CaMKII, de même que l'export nucléaire des protéines HDAC. Les régions Ras-GAP et les régions d'interaction avec la CaN de carabin agissent en synergie afin que l'inhibition du remodelage soit optimale [39]. Ainsi, favoriser l'activité de carabin, en compensant par exemple son déficit d'expression par transfert de gène, pourrait constituer une nouvelle piste pour le traitement de l'insuffisance cardiaque puisque cette protéine inhibe conjointement trois voies majeures de la signalisation conduisant à l'hypertrophie.

\section{Conclusion}

Cette revue a fait le point sur la compréhension des mécanismes mis en œuvre dans la constitution d'un remodelage cardiaque en réponse aux différents types de stress imposés au cœur. Ainsi, la protéine Epacl, 
activée par I'AMPc, semble favoriser l'hypertrophie cardiaque, la fibrose et diminuer la contractilité cardiaque dans des situations expérimentales de stress pathologiques. La protéine carabin, quant à elle, en inhibant simultanément de multiples acteurs du remodelage cardiaque pathologique ( $\mathrm{CaN}, \mathrm{CaMKII}, \mathrm{MAPK}$ ) permet de protéger les animaux de l'hypertrophie cardiaque associée à une dilatation ventriculaire et à la fibrose induite par une surcharge de pression.

Ces nouvelles connaissances pourraient offrir des opportunités pour améliorer le traitement de l'insuffisance cardiaque qui reste très imparfait malgré les progrès de ces dernières années. Les avancées considérables réalisées dans les technologies de transfert de gènes et le criblage moléculaire à haut débit pourraient faciliter le passage de ces connaissances à la clinique. $\diamond$

\section{SUMMARY}

Molecular determinants of pathological cardiac remodeling:

the examples of Epac and Carabin

Physical exercise or hypertension requires that the heart increases its hemodynamic work. However, this adaptation is based on distinct cardiac remodelling according to the physiological or pathological origin of the stress. As shown here with two examples, understanding the molecular events leading to cardiac remodeling may offer new opportunities for the development of therapies for heart failure. The recently described Epacl protein is an effector of the second messenger cAMP. Following a pathological stress, the CAMP-binding protein Epacl induces cardiac hypertrophy and fibrosis as well as alteration of calcium cycling suggesting that Epacl pharmacological inhibition may be of therapeutic value. Furthermore, the protein carabin is an important regulator of several effectors of pathological cardiac remodelling. Experimental manipulation of carabin expression profoundly alters the development of heart failure. $\diamond$

\section{REMERCIEMENTS}

Ce travail est soutenu par l'Inserm, l'université Toulouse III Paul Sabatier, la région Midi-Pyrénées, l'Association française contre les Myopathies et la Fondation pour la recherche médicale.

\section{LIENS D'INTÉRÊT}

Les auteurs déclarent n'avoir aucun lien d'intérêt concernant les données publiées dans cet article.

\section{RÉFÉRENCES}

1. Kemi 0J, Haram PM, Loennechen JP, et al. Moderate versus high exercise intensity: differential effects on aerobic fitness, cardiomyocyte contractility, and endothelial function. Cardiovasc Res $2005 ; 67: 161-72$

2. Katz AM. Cardiomyopathy of overload. A major determinant of prognosis in congestive heart failure. $N$ Engl J Med 1990 ; 322 : 100-10.

3. Levy D, Garrison RJ, Savage DD, et al. Prognostic implications of echocardiographically determined left ventricular mass in the Framingham Heart Study. N Engl J Med 1990 ; 322 : 1561-6.

4. Zannad F, Alla F, Dousset B, et al. Limitation of excessive extracellular matrix turnover may contribute to survival benefit of spironolactone therapy in patients with congestive heart failure: insights from the randomized aldactone evaluation study (RALES). Rales investigators. Circulation $2000 ; 102: 2700-6$.

5. Swynghedauw B. Molecular mechanisms of myocardial remodeling. Physiol Rev $1999 ; 79: 215-62$.

6. Molkentin JD, Lu JR, Antos CL, et al. A calcineurin-dependent transcriptional pathway for cardiac hypertrophy. Cell $1998 ; 93: 215-28$.

7. Passier R, Zeng H, Frey $\mathrm{N}$, et al. CaM kinase signaling induces cardiac hypertrophy and activates the MEF2 transcription factor in vivo. J Clin Invest $2000 ; 105$ : 1395-406.
8. Loyer X, Gómez AM, Milliez P, et al. Cardiomyocyte overexpression of neuronal nitric oxide synthase delays transition toward heart failure in response to pressure overload by preserving calcium cycling. Circulation $2008 ; 117: 3187-98$.

9. Koitabashi N, Danner T, Zaiman AL, et al. Pivotal role of cardiomyocyte TGF- $\beta$ signaling in the murine pathological response to sustained pressure overload. J Clin Invest 2011 ; 121 : 2301-12.

10. Berlo JH van, Maillet M, Molkentin JD. Signaling effectors underlying pathologic growth and remodeling of the heart. J Clin Invest 2013; 123 : 37-45.

11. Maillet M, van Berlo JH, Molkentin JD. Molecular basis of physiological heart growth: fundamental concepts and new players. Nat Rev Mol Cell Biol 2013 ; $14: 38-48$.

12. Movsesian MA, Bristow MR. Alterations in CAMP-mediated signaling and their role in the pathophysiology of dilated cardiomyopathy. Curr Top Dev Biol $2005 ; 68: 25-48$.

13. Bristow MR. Treatment of chronic heart failure with $\beta$-adrenergic receptor antagonists: a convergence of receptor pharmacology and clinical cardiology. Circ Res 2011 ; 109 : 1176-94.

14. Perrino C, Naga Prasad S V, Schroder JN, et al. Restoration of betaadrenergic receptor signaling and contractile function in heart failure by disruption of the betaARK1/phosphoinositide 3-kinase complex. Circulation $2005 ; 111: 2579-87$.

15. Métayé T, Perdrisot R, Kraimps JL. GRK et arrestines : la piste thérapeutique? Med Sci (Paris) $2006 ; 22: 537-43$.

16. Berthouze M, Laurent AC, Breckler M, et al. New perspectives in CAMPsignaling modulation. Curr Heart Fail Rep 2011 ; 8 : 159-67.

17. Mika D, Leroy J, Fischmeister R, et al. Rôle des phosphodiestérases des nucléotides cycliques de types 3 et 4 dans le couplage excitationcontraction et les arythmies cardiaques. Med Sci (Paris) $2013 ; 29: 617-22$.

18. Rooij J de, Zwartkruis FJ, Verheijen MH, et al. Epac is a Rapl guaninenucleotide-exchange factor directly activated by cyclic AMP. Nature 1998 ; $396: 474-7$.

19. Kawasaki H, Springett GM, Mochizuki N, et al. A family of cAMP-binding proteins that directly activate Rapl. Science 1998 ; 282 : 2275-9.

20. Rehmann H, Schwede F, Døskeland SO, et al. Ligand-mediated activation of the cAMP-responsive guanine nucleotide exchange factor Epac. J Biol Chem $2003 ; 278: 38548-56$.

21. Enserink JM, Christensen AE, Rooij J De, et al. A novel Epac-specific cAMP analogue demonstrates independent regulation of Rapl and ERK. Nat Cell Biol 2002; 4 : 901-6.

22. Morel $\varepsilon$, Marcantoni A, Gastineau M, et al. cAMP-binding protein Epac induces cardiomyocyte hypertrophy. Circ Res $2005 ; 97: 1296-304$.

23. Métrich M, Lucas A, Gastineau M, et al. Epac mediates beta-adrenergic receptor-induced cardiomyocyte hypertrophy. Circ Res $2008 ; 102: 959-65$.

24. Courilleau D, Bisserier M, Jullian JC, et al. Identification of a tetrahydroquinoline analog as a pharmacological inhibitor of the CAMPbinding protein Epac. J Biol Chem 2012 ; 287 : 44192-202.

25. Bisserier M, Blondeau JP, Lezoualc'h F. Epac proteins: specific ligands and role in cardiac remodelling. Biochem Soc Trans $2014 ; 42: 257-64$.

26. Métrich $M$, Laurent $A C$, Breckler $M$, et al. Epac activation induces histone deacetylase nuclear export via a Ras-dependent signalling pathway. Cell Signal $2010 ; 22$ : 1459-68.

27. Pereira L, Ruiz-Hurtado G, Morel $\varepsilon$, et al. Epac enhances excitationtranscription coupling in cardiac myocytes. J Mol Cell Cardiol 2012 ; 52 : 283-91.

28. Berthouze-Duquesnes $M$, Lucas $A$, Saulière $A$, et al. Specific interactions between Epacl, $\beta$-arrestin2 and PDE4D5 regulate $\beta$-adrenergic receptor subtype differential effects on cardiac hypertrophic signaling. Cell Signal $2013 ; 25: 970-80$.

29. Pereira L, Cheng H, Lao DH, et al. Epac2 mediates cardiac $\beta 1$-adrenergicdependent sarcoplasmic reticulum $\mathrm{Ca}^{2+}$ leak and arrhythmia. Circulation $2013 ; 127: 913-22$.

30. Okumura S, Fujita T, Cai W, et al. Epacl-dependent phospholamban phosphorylation mediates the cardiac response to stresses. J Clin Invest 2014 ; 124 : 2785-801.

31. Laurent AC, Bisserier M, Lucas A, et al. Exchange protein directly activated by cAMP 1 promotes autophagy during cardiomyocyte hypertrophy. Cardiovasc Res 2015 ; 105 : 55-64.

32. Lacampagne A, Fauconnier J, Richard S. Récepteur de la ryanodine et dysfonctionnement myocardique. Med Sci (Paris) 2008 ; 24 : 399-405.

33. Pereira L, Métrich M, Fernández-Velasco $M$, et al. The cAMP binding protein Epac modulates $\mathrm{Ca}^{2+}$ sparks by a $\mathrm{Ca}^{2+} /$ calmodulin kinase signalling pathway in rat cardiac myocytes. J Physiol $2007 ; 583: 685-94$. 


\section{RÉFÉRENCES}

34. Oestreich EA, Malik S, Goonasekera SA, et al. Epac and phospholipase Cepsilon regulate $\mathrm{Ca}^{2+}$ release in the heart by activation of protein kinase Cepsilon and calcium-calmodulin kinase II.J Biol Chem $2009 ; 284$ : 1514-22.

35. Hothi SS, Gurung IS, Heathcote JC, et al. Epac activation, altered calcium homeostasis and ventricular arrhythmogenesis in the murine heart. Pflugers Arch 2008 ; $457: 253-70$.

36. Leenders JJ, Pinto YM, Creemers દદ. Tapping the brake on cardiac growth-endogenous repressors of hypertrophic signaling. J Mol Cell Cardiol $2011 ; 51: 156-67$.

37. Pan F, Sun L, Kardian DB, et al. Feedback inhibition of calcineurin and Ras by a dual inhibitory protein Carabin. Nature 2007 ; $445:$ 433-6.

38. Schickel JN, Pasquali JL, Soley A, et al. Carabin deficiency in B cells increases BCR-TLR9 costimulation-induced autoimmunity. EMBO Mol Med $2012 ; 4$ : 1261-75.
39. Bisserier M, Berthouze-Duquesnes M, Breckler M, et al. Carabin protects against cardiac hypertrophy by blocking calcineurin, Ras, and $\mathrm{Ca}^{2+} /$ calmodulin-dependent protein kinase II signaling. Circulation 2015; 131 : 390-400.

40. Lyon RC, Zanella F, Omens JH, et al. Mechanotransduction in cardiac hypertrophy and failure. Circ Res $2015 ; 116$ : 1462-76.

41. Pinet F, Bauters C. Potentiel des ARN non-codants comme biomarqueurs dans l'insuffisance cardiaque. Med Sci (Paris) 2015 ; 31 : 770-6.

\section{TIRÉS À PART}

F. Lezoualc'h

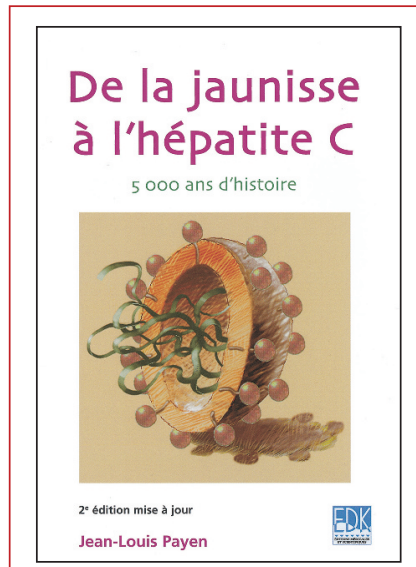

ISBN : 978-2-8425-4136-1 128 pages a jaunisse est un symptôme facilement identifiable ; il paraissait bien naturel que l'homme, confronté
à une modification de la couleur de ses yeux et de sa peau ait de tous temps recherché les causes de
cette transformation.

II n'est donc pas surprenant que le premier traité de médecine, écrit 3000 ans avant J.C. par un médecin sumérien, décrive déjà la jaunisse. À chaque époque de l'histoire de la médecine, les praticiens, influencés par les concepts médicaux de leur temps, attribuèrent une ou plusieurs explications particulières à ce symptôme. Ainsi, du démon Ahhâzu des Sumériens à la sophistication des biotechnologies qui permirent la découverte du virus de l'hépatite $C$, le lecteur cheminera sur une période de 5000 ans au travers des différents continents. Ici encore, l'histoire se révèle une formidable source de réflexion : le foie souvent impliqué dans l'apparition des jaunisses est-il le siège de l'âme?

Les expérimentations humaines chez des volontaires ou chez des enfants handicapés mentaux étaient-elles justifiées pour permettre la découverte des virus des hépatites?

Le formidable développement de la transfusion sanguine, des vaccinations, mais aussi de la toxicomanie explique-t-il les épidémies d'hépatites du XXe siècle?

Autant de questions qui sont abordées dans ce livre passionnant et accessible à tous.

\section{À retourner à EDK, 109, avenue Aristide Briand - 92541 Montrouge Cedex}

UU Tél. : 0141177405 - Fax : 0143293262 - E-mail : edk@edk.fr

NOM :

Prénom :

Adresse :

Code postal :

Ville :

Pays :

Fonction :

Je souhaite recevoir l'ouvrage De la jaunisse à l'hépatite $\mathbf{C , ~} \mathbf{5} 000$ ans d'histoire : $12 €+3 €$ de port $=\mathbf{1 5} € \mathbf{T T C}$

en ................ exemplaire, soit un total de $€$

U $\square$ Par chèque, à l'ordre de $\mathbf{E} \mathbf{D} \mathbf{K}$

$\square$ Par carte bancaire : $\square$ Visa $\square$ Eurocard/Mastercard

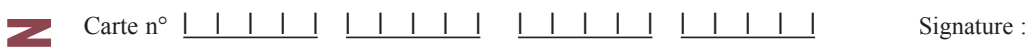

$\odot$ Date d'expiration: $\quad 1 \quad 1 \quad 1 \quad 1 \quad 1$

- $\mathrm{N}^{\circ}$ de contrôle au dos de la carte : | | | |

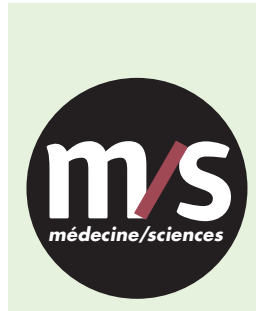

Tarifs d'abonnement $m / s-2015$

$>$ Grâce à $m / s$, vivez en direct les progrès des sciences biologiques et médicales

Abonnez-vous

à médecine/sciences

Bulletin d'abonnement page 933 dans ce numéro de $\mathrm{m} / \mathrm{s}$

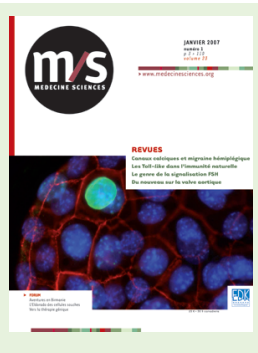

